\title{
PSEUDOMONAS STUTZERI IN PATHOLOGICAL MATERIAL
}

\author{
S. P. Lapage, L. R. Hill and Jeanne D. Reeve \\ National Collection of Type Cultures, Central Public Health Laboratory, Colindale, and \\ Department of Pathology, Prince of Wales General Hospital, London
}

\section{Plates XV AND XVI}

SIX strains of Pseudomonas, isolated from four patients in the United Kingdom, have been identified as Pseudomonas stutzeri. No previous isolations of this organism from human sources in this country have been reported and it appears to be a little-known species.

The organism known as Pseudomonas stutzeri (Lehmann and Neumann) Kluyver (1942) was first described by Burri and Stutzer (1895) under the name Bacillus denitrificans II. It is of wide distribution in nature in manure, humus, straw, pond water, sewage and similar situations (Jensen, 1898; Höflich, 1902; Van Iterson, 1902, cited by Höflich). The classification and recognition of the organism were confused until van Niel and Allen's paper in 1952, which gave a detailed account of the nomenclatural changes the organism has undergone. Since then, several isolations from human sources have been described. Wetmore and Gochenour (1956) mentioned one strain of human origin which they investigated. King (1964) mentioned 14 strains from blood cultures, 10 from cerebrospinal fluid, 12 respiratory, 5 urinary, 2 faecal, 3 genital, 5 ear, 2 eye, and 13 from miscellaneous infections. Von Graevenitz (1965) described 4 strains from clinical material. Two strains were from patients with malignant tumours, a further strain from an infected bursa and the last strain was from a case of collapse of lung due to a foreign body. Two of these patients were aged and in three of the cases other bacterial pathogens were also present. Von Graevenitz suggested that Ps. stutzeri may live as a saprophyte in the human body and cause serious infection if the general defence mechanisms of the patient have been weakened.

Stanier, Palleroni and Doudoroff (1966) described 17 strains of Ps. stutzeri that they obtained from laboratories in Denmark and France, originally from clinical cases. Mandel (1966) reported that the guanine and cytosine contents (per cent. GC) of DNA extracted from these strains fell into two groups: $62 \cdot 1 \pm 1$ per cent. GC and $65 \pm 1$ per cent. GC respectively. The base compositions of DNA from six strains of $P$ s. pseudomallei were $69.5 \pm 0.7$ per cent. GC, whilst the range of values for the genus Pseudomonas was 58-70 per cent. GC.

The purpose of the present paper is to report the characteristics of the six Ps. stutzeri strains we have examined. We have compared this species with Ps. pseudomallei, with which it may be confused as the two organisms give the same results in a number of common biochemical tests and the rough colony form of Ps. stutzeri might be mistaken for Ps. pseudomallei.

\section{CASE REPORTS}

Case 1. A 69-yr-old woman was admitted to hospital with haematemesis, later fatal, said to be from a duodenal ulcer. She had chronic ulcers on both legs, an incidental finding

Received 27 Mar. 1968; accepted 22 Apr. 1968.

J. MED. MICROBIOL.-VOL. 1 (1968) 
unconnected with the haematemesis. A strain of Ps. stutzeri was isolated from the leg ulcers.

Case 2. An Indian male of $42 \mathrm{yr}$ who had been in England for $2 \mathrm{yr}$. Ps. stutzeri was isolated from cervical-node tissue taken during biopsy. The patient had had a porto-caval shunt for cirrhosis of the liver 2 yr before and later suffered from encephalopathy. A year after the shunt he developed pyrexia thought to be due to a staphylococcal chest infection. Antibiotic treatment improved the chest condition, but he remained pyrexial. He then developed a hot and swollen left ankle and right wrist, and septicaemia with arthritis was diagnosed. Three successive blood cultures proved sterile. Cervical lymphadenopathy was noticed and a biopsy of the gland yielded Ps. stutzeri, which on isolation appeared in 2 strains, "smooth" and "rough". He was later thought to be suffering from acute bacterial endocarditis and possibly rheumatic fever as his antistreptolysin-O titre rose from 130 to 570 units. A lesion developed at the upper end of the right tibia, and was believed to be osteomyelitis. He failed to respond to treatment with massive doses of a large variety of antibiotics, but on cessation of treatment the temperature settled. He was discharged from hospital and has remained reasonably well since except for recurrent attacks of hepatic pre-coma. Ps. stutzeri was isolated only once from the patient, i.e., from the cervical biopsy; 3 blood cultures and the tibial lesion were negative.

Case 3. An English female laboratory technician of $23 \mathrm{yr}$ who had recurrent infections for $6 \mathrm{mth}$ had been admitted to hospital four times. On the first three admissions the infection was a deep-seated abscess of the buttock yielding Staphylococcus aureus, and the lesion appeared to relapse on one occasion. Staph. aureus was isolated from a blood culture once. Five months after the first episode she developed mild fever and an effusion in the left knee, and her white cell blood count was 20,000 per c.mm. No bacterial growth was obtained on culture of fluid aspirated from the knee. Her temperature rose to $102^{\circ}-103^{\circ} \mathrm{F}$ and two sets of blood cultures were taken. Ps. stutzeri was isolated from all the bottles of one set and one out of three of the other set. Several other blood cultures were negative. The patient recovered and has remained apyrexial.

Case 4. Two strains were isolated from the leg ulcer of an elderly English woman, together with a mixture of Gram-negative rods. Further information is not available on this case.

\section{MATERIALS AND METHODS}

\section{Organisms}

A total of 13 strains have been examined. Six of the strains of Ps. stutzeri were from the above four cases. Two of the strains of Ps. pseudomalle $i$ were isolated from one patient.

The Ps. stutzeri strains were as follows. No. $1=\mathrm{Leg}$ ulcer strain from case $1=$ NCTC 10450. No. $2 a$ and $2 b=$ Smooth and rough strains respectively isolated from the cervical node biopsy specimen of case 2 . No. $3=$ Blood culture strain from case 3 . No. $4 \mathrm{a}$ and $4 \mathrm{~b}=$ Smooth and rough strains isolated from the leg ulcer of case 4 . No. $5=$ National Collection of Industrial Bacteria, NCIB $9040=$ NCTC 10473. No. $6=$ NCIB $9721=$ NCTC $10475=$ ATCC 11607.

The $P$ s.pseudomallei strains were: no. $1 \mathrm{a}$ and $1 \mathrm{~b}$ from pus and surgical wound respectively from a case of melioidosis. No. $2=$ NCTC 1688 . No. $3=$ NCTC 8016. No. $4=$ NCTC 10276, strain Prince, described by Maegraith and Leithead (1964).

\section{Bacteriological investigations}

The recently isolated strains had been examined both in the laboratories where they had been isolated and in the NCTC over a period of time and had not been subjected to precisely the same set of characterisation procedures. They were, therefore, all re-examined, together with the two NCIB Ps. stutzeri and three NCTC Ps. pseudomallei strains. Most of the characters here reported were therefore determined at least twice. Except where otherwise indicated, all tests were carried out at $37^{\circ} \mathrm{C}$ and examined after 24 and 48 hours' incubation. 
Microscopical examinations. Gram staining was made on films from 24-hr nutrient agar cultures. Flagellar staining was by Plimmer and Paine's (1921) method as specified in Cowan and Steel (1965). Accumulation of poly- $\beta$-hydroxybutyrate was determined by sudan black staining of 48-hr cultures grown on the chemically defined medium of Stanier et al. (1966) solidified with 1 per cent. ionagar (Oxoid).

Cultural and physiological characters. Colonial morphology and pigment production on nutrient agar were recorded after $24 \mathrm{hr}$, and again after $1 \mathrm{wk}$ at room temperature. Subcultures on media A and B of King, Ward and Raney (1954) were examined under ultraviolet (UV) illumination for pyocyanin and fluorescein pigments. Growth in nutrient broth was recorded after $24 \mathrm{hr}$; temperature ranges for growth were determined on nutrient agar slants incubated at $4^{\circ} \mathrm{C}(1 \mathrm{wk}), 22^{\circ}, 30^{\circ}, 37^{\circ}$ and $42^{\circ} \mathrm{C}(48 \mathrm{hr})$. Anaerobic growth on nutrient agar was tested for by incubation in a McIntosh and Fildes' jar. Haemolytic activity was determined on 5 per cent. horse blood agar and growth on MacConkey's agar (Ministry of Health, 1956) was recorded.

Acid production from carbohydrates. Oxidation or fermentation of glucose was determined from stab cultures in Hugh and Leifson's (1953) O-F medium. Aerobic acid production from glucose, lactose, maltose, mannitol, salicin and sucrose was tested for in peptone water media with bromcresol purple indicator. The same carbohydrates were also tested with the ammonium salt media of Smith, Gordon and Clark (1952), and, in addition, arabinose, dulcitol and sorbitol. Incubation for both series was continued up to 14 days. The strains were also stab inoculated into 10 per cent. lactose agar, five strains per petri dish.

Nitrate reduction. Two sets of nitrate broths (Cowan and Steel), containing Durham's tubes, were inoculated and incubated aerobically and anaerobically. Presence of nitrite after 3,10 and 20 days was tested on approximately $1-\mathrm{ml}$ volumes by the Griess-Ilosvay method. To tubes not giving a positive nitrite reaction, zinc dust was added to test for presence of nitrate. Gas collecting in the Durham's tubes was also recorded.

Further biochemical and other tests. The following tests were also carried out as described by Cowan and Steel. The particular method followed when alternatives are given is indicated in parenthesis: catalase; oxidase; utilisation of citrate (method 1, Koser's); KCN tolerance; production of indole (method 2); methyl-red; acetoin production (method 1, Barritt's method); hydrogen sulphide production (method 3, with nutrient broth); gelatin liquefaction (method 1); oxidation of gluconate (method 2); utilisation of malonate; phenylalanine deamination (method 2); decarboxylase reactions with arginine, lysine and ornithine (method 1, Møller, 1955); LV (lecithovitellin) reaction; urease activity (method 1, Christensen's medium); starch hydrolysis; casein digestion; presence of $\beta$-galactosidase (ONPG).

Arginine desimidase was tested for by stab inoculations of Thornley's (1960) medium. Heat resistance of alkaline phosphatases was determined by Liu's (1966) method.

\section{RESULTS}

All Pseudomonas stutzeri and Ps.pseudomallei strains possessed the following characteristics. They were Gram-negative, motile rods measuring about $0.5 \times 1 \cdot 5-3 \mu \mathrm{m}$, which grew on nutrient agar aerobically and anaerobically. Definite pigmentation was not produced on nutrient agar, nor was any fluorescence observed under ultraviolet illumination of cultures grown on the A and B media of King et al. Ring or pellicle growth with turbidity and a deposit was obtained in nutrient broth and all strains grew at temperatures ranging from $22^{\circ}$ to $37^{\circ} \mathrm{C}$. No true $\beta$-haemolysis was observed on blood agar and the strains grew as non-lactose fermenting colonies on MacConkey's agar. They gave an oxidative reaction in Hugh and Leifson's medium, using glucose as a substrate, and positive reactions in the following tests: catalase, oxidase, citrate, arginine decarboxylase (Møller's method), except 4 strains of Ps. stutzeri which grew 
very poorly in the last test. Negative reactions were obtained for indole, MR, acetoin, gluconate, phenylalanine, ONPG, and lysine and ornithine decarboxylase tests. Differences were observed, however, either among Ps. stutzeri or among Ps. pseudomallei strains or between these two species with a number of tests. These results are summarised in the table.

TABLE

Characters of Pseudomonas stutzeri and Ps. pseudomallei

\begin{tabular}{|c|c|c|c|c|c|c|c|c|c|c|c|c|c|}
\hline \multirow{3}{*}{ Character } & \multicolumn{13}{|c|}{ Presence $(+)$ or absence $(-)$ of character in } \\
\hline & \multicolumn{8}{|c|}{ Ps. stutzeri strain } & \multicolumn{5}{|c|}{ Ps. pseudomallei strain } \\
\hline & 1 & $2 \mathrm{a}$ & $2 b$ & 3 & $4 a$ & $4 b$ & 5 & 6 & $1 \mathrm{a}$ & $1 b$ & 2 & 3 & 4 \\
\hline Rough colonies. & + & - & - & - & - & + & - & - & + & + & + & + & + \\
\hline Smooth colonies & + & + & + & + & + & + & + & + & - & - & - & $\frac{t}{-}$ & I \\
\hline Metallic sheen to mass growth & - & - & - & - & - & - & - & - & + & + & + & + & + \\
\hline Growth at $42^{\circ} \mathrm{C}$ & + & + & + & + & - & - & + & + & + & + & + & + & + \\
\hline Growth at $5^{\circ} \mathrm{C}$ & - & - & - & - & + & + & - & + & - & - & - & - & - \\
\hline $\begin{array}{l}\text { Accumulation of } \beta \text {-hydroxy- } \\
\text { butyrate }\end{array}$ & - & - & - & - & - & - & - & - & + & + & + & + & + \\
\hline Acid produced* from: & & & & & & & & & & & & & \\
\hline $\begin{array}{l}\text { Glucose } \\
\text { Dulcitol, lactose, maltose, } \\
\text { sucrose }\end{array}$ & $\stackrel{+}{-}$ & \pm & \pm & \pm & \pm & \pm & \pm & $\stackrel{t}{-}$ & + & $\begin{array}{l}+ \\
+\end{array}$ & $\begin{array}{l}+ \\
+\end{array}$ & + & + \\
\hline $\begin{array}{l}\text { Mannitol : }: \\
\text { Salicin }\end{array}$ & + & - & - & - & - & - & - & + & + & + & + & + & \pm \\
\hline $\begin{array}{l}\text { Salicin } \\
\text { Acid produced from io per cent. } \\
\text { lactose agar }\end{array}$ & $\overline{-}$ & $\overline{-}$ & $\overline{-}$ & $\overline{-}$ & - & $\overline{-}$ & $\overline{-}$ & $\overline{-}$ & $\bar{t}$ & $\overline{+}$ & $\overrightarrow{+}$ & $\bar{t}$ & $\overline{+}$ \\
\hline Nitrites reduced. & + & + & + & + & + & + & + & + & - & - & - & - & - \\
\hline $\mathrm{KCN}$ tolerance & - & - & - & - & - & - & - & - & + & + & + & + & + \\
\hline $\mathrm{H}_{2} \mathrm{~S}$ production & - & d & $\mathrm{d}$ & d & - & - & + & - & - & - & - & - & - \\
\hline Gelatin liquefaction . & - & - & - & - & - & - & - & - & + & + & + & + & + \\
\hline Opalescence on LV agar & - & - & - & - & - & - & - & - & + & + & + & + & + \\
\hline Urease ( 5 days): & + & - & + & - & - & + & + & - & - & - & $\bar{d}$ & - & - \\
\hline Starch hydrolysis & + & + & + & + & + & + & + & + & - & - & $\mathrm{d}$ & $\mathrm{d}$ & d \\
\hline Casein hydrolysis . & - & - & - & - & - & - & - & - & + & + & + & + & + \\
\hline Arginine desimidase . & - & - & - & - & - & - & - & - & + & + & + & + & + \\
\hline Heat-stable alkaline phosphatase & - & - & - & - & - & - & - & - & + & + & + & + & + \\
\hline
\end{tabular}

* On the ammonium salt medium of Smith et al. (1952). $\mathrm{d}=$ Results of doubtful significance.

\section{Pseudomonas stutzeri}

Microscopically, all eight strains were found to be motile by a single polar flagellum. They did not accumulate poly- $\beta$-hydroxybutyrate as an intracellular reserve material.

Two main types of colony were observed on nutrient agar: a dry, coherent, ridged colony (" rough ") with concentric spreading areas and a mucoid colony (" smooth ") together with various intermediate types. The range of colonies obtained is illustrated in figs. 1 and 2 . The variety in colonial appearance is such that some cultures of Ps. stutzeri could be mistaken for mixed cultures by observers not familiar with the organism. Six strains (no. 2a, 2b, 3, 4a, 
5 and 6) were of the smooth type, although $2 b$ had been regarded at first as a rough strain. Strains 1 and $4 \mathrm{~b}$ continually dissociated into both types. This pattern could be discerned after 24 hours' incubation, but was more marked after $1 \mathrm{wk}$ at room temperature. Strains $1,2 \mathrm{a}, 2 \mathrm{~b}, 3$ and 5 grew at $42^{\circ} \mathrm{C}$, but not at $5^{\circ} \mathrm{C}$, strains $4 \mathrm{a}$ and $4 \mathrm{~b}$ grew at $5^{\circ} \mathrm{C}$, but not at $42^{\circ} \mathrm{C}$, whilst strain 6 grew at both temperatures.

In peptone water media all strains produced acid from glucose; only a few weak acid reactions from other carbohydrates were recorded: lactose (strain 5), mannitol (strains 1, 2a, 5 and 6 ) and sucrose (strains $2 \mathrm{~b}$ and 6 ). No acid reactions were recorded for maitose or salicin. On ammonium salt media, acid was produced from glucose but not from dulcitol, salicin or sucrose. No acid was produced within 7 days from lactose, maltose or mannitol, but some strains produced weak acid of doubtful significance from these carbohydrates after 14 days' incubation. One strain (no. 5) grew very poorly on these media. No acid was produced on 10 per cent. lactose agar.

The production of gas in nitrate media aerobically and anaerobically is characteristic of Ps. stutzeri. The speed with which it was produced varied from strain to strain and from one test to another. However, aerobically all strains had by the 3 rd day produced copious amounts of gas and no nitrite could be detected in the medium. Anaerobically, gas production was slower, but definite by the 5 th day (except for strains 1 and $4 \mathrm{~b}$ which required incubation up to 10 days). Incubation was prolonged to 20 days for complete reduction. The frothing appearance of the nitrate broths (Van Niel and Allen) was more noticeable under aerobic conditions and was very marked, except for strains $4 \mathrm{a}$ and $4 \mathrm{~b}$.

All eight strains failed to grow in $\mathrm{KCN}$ broth. $\mathrm{H}_{2} \mathrm{~S}$ production, as detected by lead acetate paper over nutrient broth cultures, gave variable results upon repetition. None of the strains liquefied gelatin within $3 \mathrm{wk}$, only five utilised malonate and all grew on lecithovitellin agar without zones of opalescence. Four strains (no. 1, 2b, 4b and 5) gave positive results in Christensen's (1946) urea medium in 5 days, two strains ( $2 \mathrm{a}$ and 3 ) were positive after 14 days and the remaining two strains were doubtfully positive after 14 days. All strains hydrolysed starch although one strain (no. 5) gave variable results upon repetition. None of them hydrolysed casein or produced a desimidase for arginine (Thornley's method). The alkaline phosphatases were heat labile, for they were inactivated by heating to $70^{\circ} \mathrm{C}$ for $20 \mathrm{~min}$.

\section{Pseudomonas pseudomallei}

The five strains of $P$ s. pseudomallei showed multitrichous polar flagella and intracellular deposits of poly- $\beta$-hydroxybutyrate were evident.

They were colonially homogeneous; the dry, coherent colonies (" rough ") produced on nutrient agar are illustrated in figs. 3 and 4 (see also fig. 5). In contrast with $P$ s. stutzeri, they were all of a single type and were distinguishable on close examination from the rough colonies of Ps. stutzeri. All strains grew at $42^{\circ}$ but not at $5^{\circ} \mathrm{C}$. 
In the peptone water media four produced acid from glucose (strain 1a was not tested). In addition, strains $1 \mathrm{~b}$ and 3 produced acid from lactose, strain 3 from maltose, and strain $1 \mathrm{~b}$ from mannitol. No acid reactions were recorded for salicin or sucrose. With the ammonium salt media the Ps. pseudomallei strains were more active than the Ps. stutzeri strains. Acid was produced from arabinose, dulcitol, lactose, maltose, mannitol, sorbitol and sucrose. Only one strain (no. 3) produced acid from salicin after 14 days' incubation. They all produced acid on 10 per cent. lactose agar in 5 days.

None of the Ps. pseudomallei strains produced gas in the nitrate broth, incubated aerobically or anaerobically; they reduced nitrate to nitrite within 2 days, but even after 20 days' incubation the nitrites were not further reduced.

They all grew in KCN broth and did not produce $\mathrm{H}_{2} \mathrm{~S}$. They liquefied gelatin within 2-3 days. One strain only (no. 1a) utilised malonate, but they all grew on lecithovitellin agar with evident zones of opalescence. None of them produced urease in 5 days, but after 14 days strains 1a, 2 and 4 gave weak positive reactions. Strains $1 \mathrm{a}$ and $1 \mathrm{~b}$ were consistently negative with the starch hydrolysis test, strains 2, 3 and 4 have been read as negative on four occasions and positive on one occasion. They all hydrolysed casein and gave positive reactions in Thornley's test for arginine desimidase. Their alkaline phosphatases were heat stable.

\section{Discussion}

The characters of the six Pseudomonas stutzeri strains recently isolated from patients agree with those of the two NCIB strains and with those given by Burri and Stutzer (1895) and van Niel and Allen (1952), and by Stanier et al. (1966), who refer to two outstanding properties of Ps. stutzeri that have traditionally served to identify it: its remarkable denitrifying ability and its unusual colony structure.

The denitrifying ability of our strains was very marked. Stanier et al. found that their smooth strains were less active denitrifiers anaerobically than their rough strains. We could not confirm this. Anaerobically, two smooth strains (no. 3 and 5) were quickest in producing gas (2 days) whereas our two rough strains (no. 1 and $4 \mathrm{~b}$ ) did not produce gas until the 10th day of incubation. However, aerobic results with the nitrate test give a quick and certain distinction between Ps. stutzeri and Ps. pseudomallei. With the former, frothing of the broths was evident after only 24 hours' incubation and by the 3 rd day copious amounts of gas were collected in the Durham's tubes, the nitrate being completely reduced. By contrast, Ps. pseudomallei, though actively reducing nitrates to nitrites, does not further reduce the nitrites and no gas is therefore produced.

No reliance for the identification of strains from human sources can be placed on the colonial appearance of Ps. stutzeri. Most of the strains used by Stanier $e t a l$. were smooth, but they had been received from other laboratories. On fresh isolation from human sources both colony forms may be found, with perhaps the smooth form predominating. Stanier et al. state that several of their smooth strains regained the rough colony form, and greater denitrifying 
ability, by one or two passages through denitrification media. We have not investigated this point thoroughly; only one series of subcultures was made without a change in colony form.

van Niel and Allen state that anaerobic growth of Ps. stutzeri is possible only in media containing nitrate, nitrite or nitramide, or if exposed to $\mathrm{N}_{2} \mathrm{O}$. All our strains grew anaerobically on nutrient agar in a McIntosh and Fildes' jar. There may have been traces of nitrate in our nutrient agar, but we could not detect any in the Oxoid nutrient base using Griess-Ilosvay reagents and zinc.

Starch hydrolysis gave variable results. Ps. stutzeri strains were consistently positive except for one strain which gave variable results. The two recent clinical isolations of $P$ s. pseudomalle $i$ were consistently negative, but the National Collection strains gave variable results. The discrepancies may have been due to compositional differences in different batches of our starch agar and show that too great reliance should not be placed on this test.

It can be seen that the identification of Ps. stutzeri is not difficult provided that the possibility of its occurrence in human infections is borne in mind. Colonially, even when rough, the $P$ s. stutzeri strains can be distinguished from the Ps. pseudomallei strains. Ps. stutzeri is motile by a single polar flagellum, whereas Ps. pseudomallei possesses multitrichous polar flagella. Ps. pseudomallei possesses heat-stable alkaline phosphatases, liquefies gelatin promptly, produces an arginine desimidase, accumulates poly- $\beta$-hydroxybutyrate intracellularly, hydrolyses casein, grows in potassium cyanide media, and produces opalescent zones on lecithovitellin agar. None of these properties is shared with Ps. stutzeri. In addition, Ps. pseudomallei produces acid from more carbohydrates than $P$ s. stutzeri, especially on ammonium salt media, and it produces acid on 10 per cent. lactose agar, whereas $P$ s. stutzeri does not.

We agree with Von Graevenitz (1965) that it is difficult to assess the pathogenicity of Ps. stutzeri. In cases 1 and 4 it was isolated from chronic leg ulcers and may well have been a saprophyte. In case 2 the isolation was from a cervical gland biopsy of a patient in poor general health, and in case 3 the isolation of the organism was from the blood culture of a patient with recurrent pyrexial episodes possibly due to Staphylococcus aureus. In cases 2 and 3 Ps. stutzeri may have been a secondary invader, when the general resistance of the patient was lowered. Dr Nuala Crowley tells us that she would not attribute any pathogenic role to the strains from cases 2,3 and 4 , which were isolated in her laboratory.

\section{SUMMARY}

Six strains of Ps. stutzeri, recently isolated from pathological material obtained from human sources in this country, as well as two strains from the National Collection of Type Cultures, were compared with five strains of Ps. pseudomallei.

The two species could readily be differentiated by means of the aerobic nitrate reduction test: Ps. stutzeri reduces nitrate rapidly and completely with the production of gas, whereas $P$ s. pseudomallei reduces nitrate only to nitrite. Ps. stutzeri has a single polar flagellum, Ps. pseudomallei has several polar 
flagella. Ps. pseudomallei has heat-stable alkaline phosphatases and gives positive results in the tests for gelatin liquefaction, arginine desimidase, intracellular accumulation of poly- $\beta$-hydroxybutyrate, casein hydrolysis, $\mathrm{KCN}$ tolerance and opalescence on LV agar. Ps. stutzeri strains were negative in all these respects.

At present it is not possible to say for certain whether Ps. stutzeri found in pathological material represents a secondary invader in a debilitated host or should be regarded as a contaminant.

We would like to express our gratitude to Dr Nuala Crowley and Dr L. Robertson, who provided the freshly isolated strains; to Dr J. M. Shewan who provided the two NCIB strains of Ps. stutzeri; to Professor Sheila Sherlock for permission to publish her cases; and to Mrs G. Waters and Mr J. J. S. Snell for excellent technical assistance.

\section{REFERENCES}

Burri, R., ANd Stutzer, A. $\quad$ - $\quad$ 1895. Zentbl. Bakt., Abt. II, 1, 257, 350, 392

Christensen, W. B. . . . . . $\quad$. 1946. J. Bact., 52, 461.

CowAN, S. T., AND SteEl, K. J. - 1965. Manual for the identification of medical

HöFLICH, C. bacteria, London, pp. 150-165.

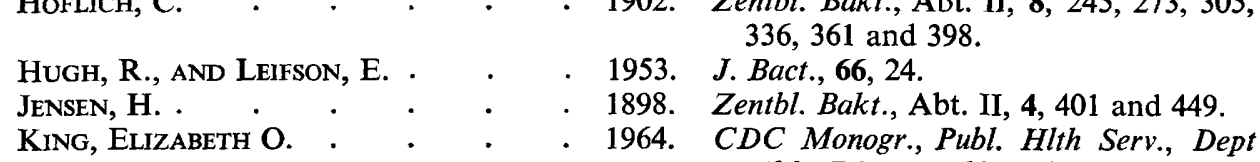

King, Elizabeth O., Ward, Martha K., 1954. J. Lab. Clin. Med., 44, 301. AND RANEY, D. E.

Liu, P. V. - . . . . . . 1966. Amer. J. Clin. Path., 45, 639.

MaEgraith, B. G., AND Leithead, C. S. 1964. Lancet, 1, 862.

MANDEL, M. . . . . . 1966. J. Gen. Microbiol., 43, 273.

Ministry of Health . . . . 1956. Rep. Publ. Hlth Med. Subj., Lond., no. 71,3 rd ed.

Møller, V. . . . . . . 1955. Acta path. microbiol. scand., 36, 158.

Plimmer, H. G., and Paine, S. G.. 1921. J. Path. Bact., 24, 286.

Smith, N. R., Gordon, R. E., ANd Clark, 1952. Agriculture Monogr., no. 16.

F. E.

Stanier, R. Y., Palleroni, N. J., AND 1966. J. Gen. Microbiol., 43, 159.

DOUDOROFF, $M$.

ThORNLEY, M. J. $\quad$. . $\quad$. $\quad$. 1960. J. Appl. Bact., 23, 37.

VAN Niel, C. B., AND Allen, M. B. . 1952. J. Bact., 64, 413.

Von Graevenitz, A. . . . . . 1965. Amer. J. Clin. Path., 43, 357.

Wetmore, Psyche W., AND Gochenour, 1956. J. Bact., 72, 79.

W. S., JR 
PSEUdOMONAS STUTZERI AND PS. PSEUdOMALLEI

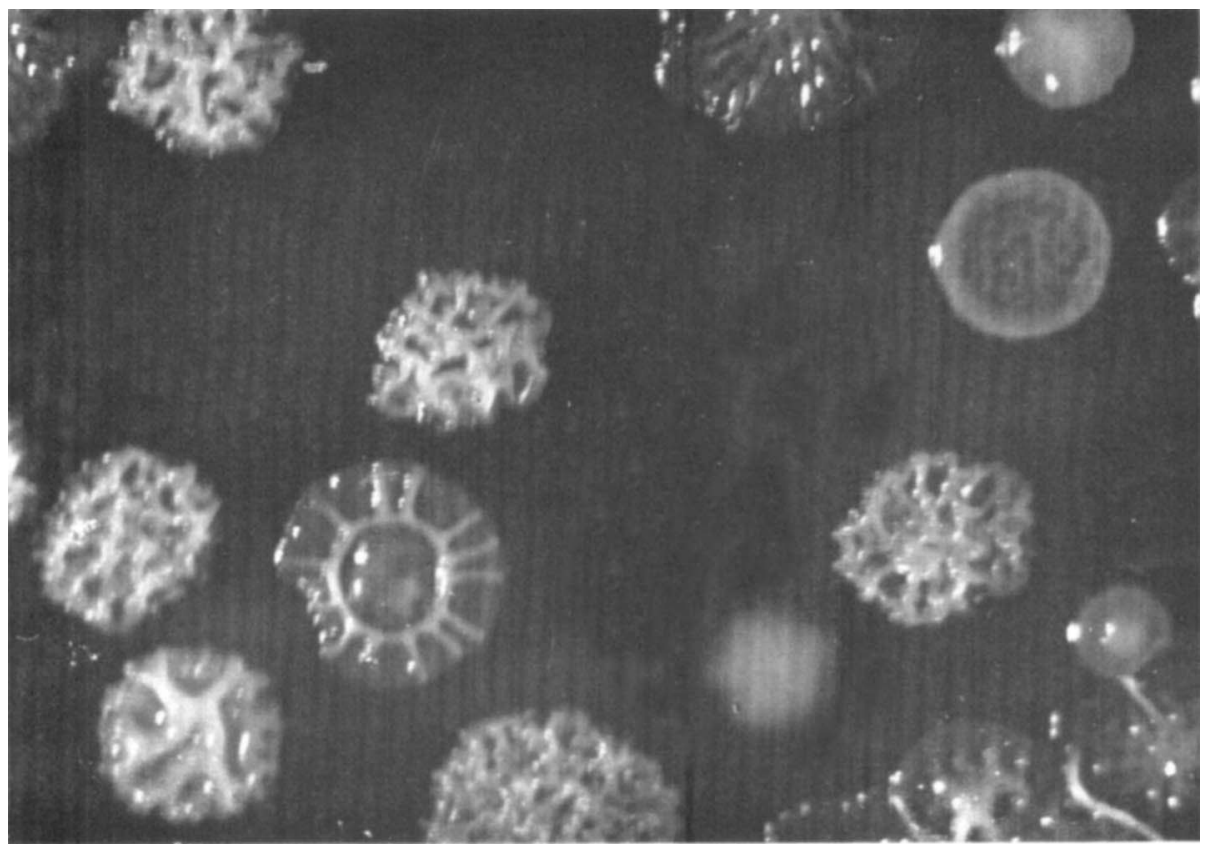

Fig. 1.-Pseudomonas stutzeri, grown for $48 \mathrm{hr}$ at $37^{\circ} \mathrm{C}$ and left at room temperature for further 3 days. Oblique light. $\times 9$.

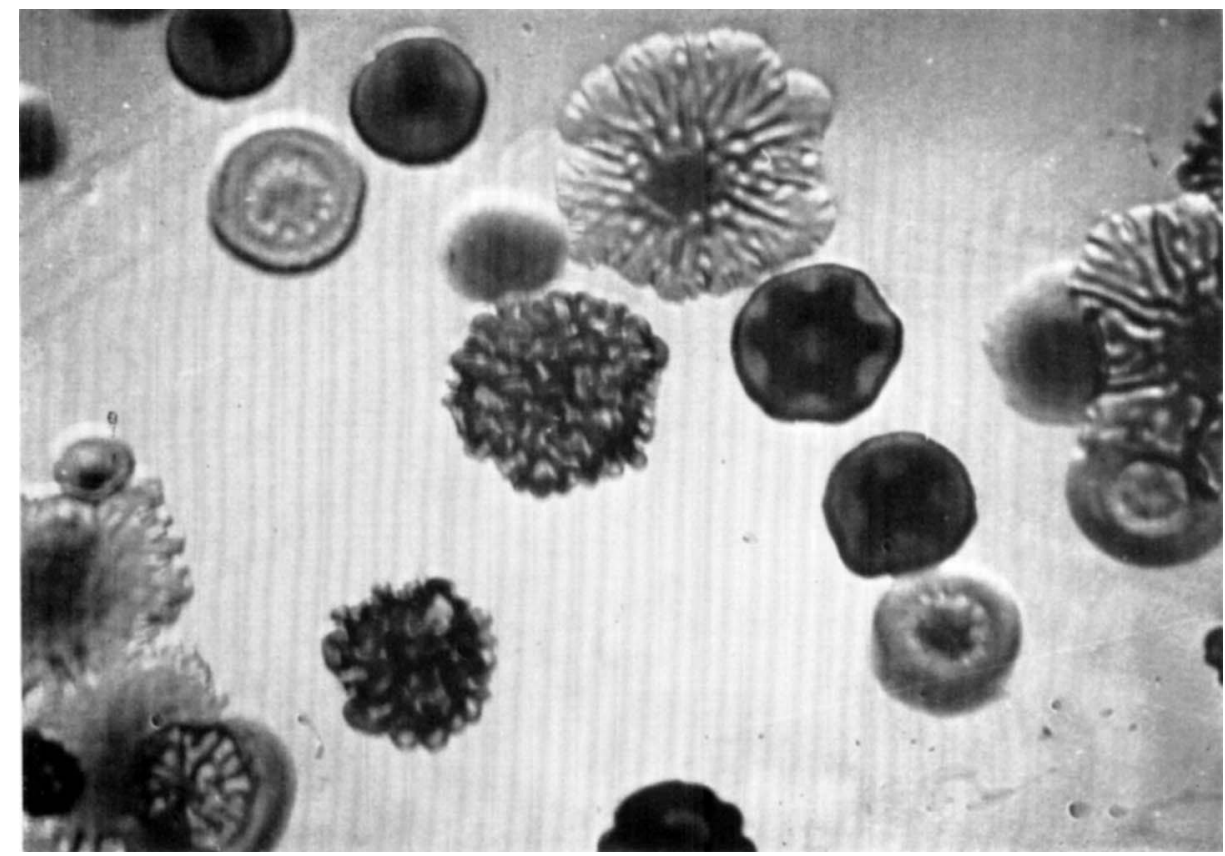

FIG. 2.-Ps. stutzeri, as fig. 1. Transmitted light. $\times 9$. 
PSEUDOMONAS STUTZERI AND PS. PSEUDOMALLEI

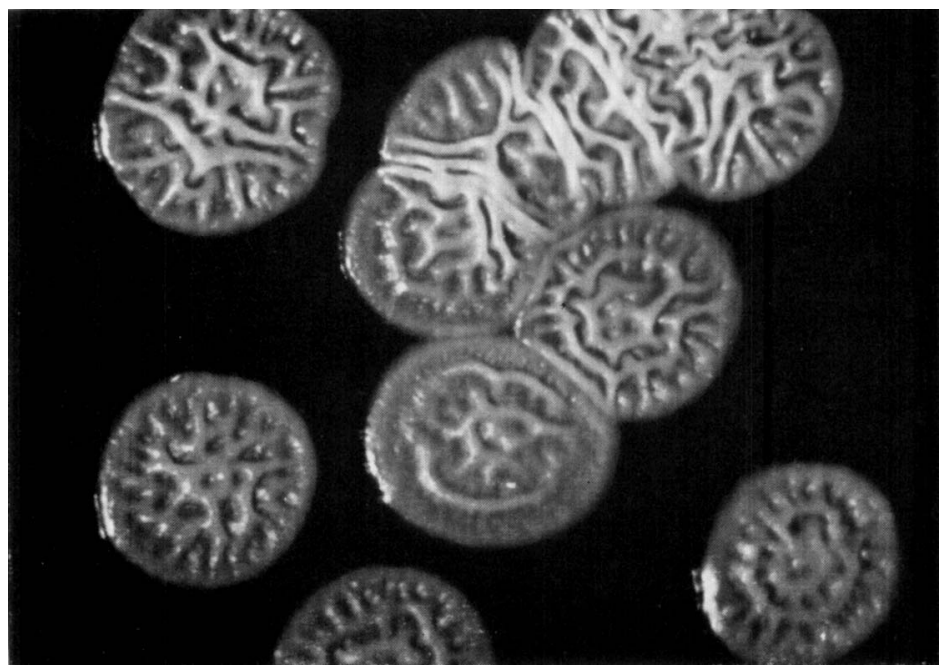

Fig. 3.-Ps. pseudomallei, grown for $48 \mathrm{hr}$ at $37^{\circ} \mathrm{C}$ and left at room temperature for further 3 days. Oblique'light. $\times 7$.

FIG. 4.-Ps. pseudomallei, as fig. 3. Transmitted light. $\times 7$.
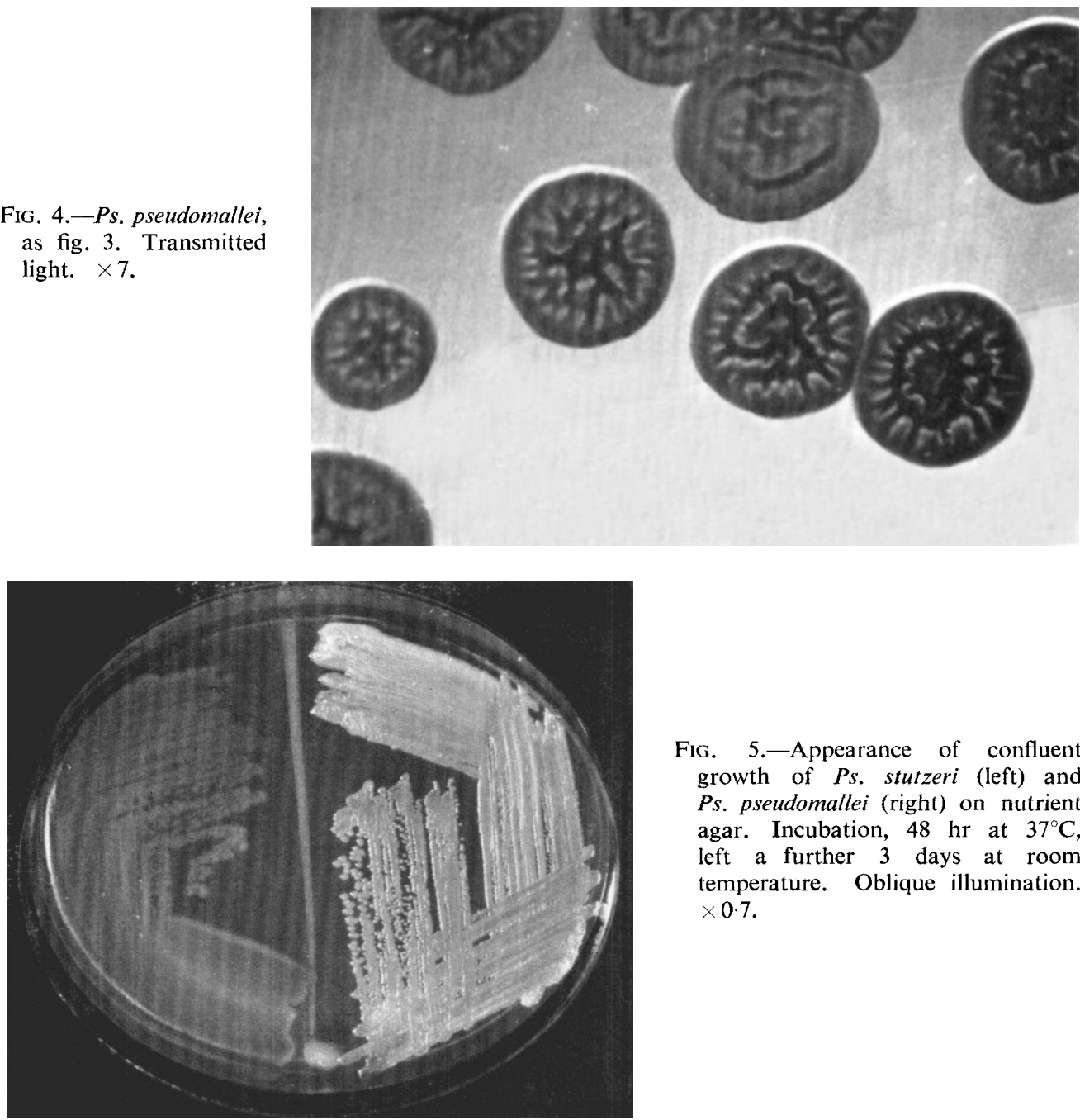

FIG. 5.-Appearance of confluent growth of Ps. stutzeri (left) and Ps. pseudomallei (right) on nutrient agar. Incubation, $48 \mathrm{hr}$ at $37^{\circ} \mathrm{C}$, left a further 3 days at room temperature. Oblique illumination. $\times 0.7$. 